Published in final edited form as:

Semin Cancer Biol. 2020 October ; 65: 91-98. doi:10.1016/j.semcancer.2019.12.002.

\title{
Mechanisms of resistance to CAR T cell therapies
}

\author{
Nathan Singh ${ }^{1}$, Elena Orlando², Jun $\mathrm{Xu}^{3}$, Jie $\mathrm{Xu}^{3}, \mathrm{Zev}$ Binder ${ }^{4}, \mathrm{McKensie} \mathrm{A.} \mathrm{Collins}^{3}$, \\ Donald M. O'Rourke ${ }^{4}$, J. Joseph Melenhorst ${ }^{3,{ }^{*}}$ \\ ${ }^{1}$ Division of Oncology, Section of Stem Cell Biology, Washington University School of Medicine, \\ St. Louis, Missouri, 63105 \\ ${ }^{2}$ Novartis Institutes for Biomedical Research, Cambridge, Massachusetts, 02139 \\ ${ }^{3}$ Department of Pathology and Laboratory Medicine, Perelman School of Medicine at the \\ University of Pennsylvania, Philadelphia, Pennsylvania, 19104 \\ ${ }^{4}$ Department of Neurosurgery, Perelman School of Medicine at the University of Pennsylvania, \\ Philadelphia, Pennsylvania, 19104
}

\begin{abstract}
Chimeric antigen receptor (CAR)-engineered $\mathrm{T}$ cells have demonstrated remarkable success in the treatment of B cell malignancies. FDA approval of these therapies represents a watershed moment in the development of therapies for cancer. Despite the successes of the last decade, many patients will unfortunately not experience durable responses to CAR therapy. Emerging research has shed light on the biology responsible for these failures, and further highlighted the hurdles to broader success. Here, we review the recent research identifying how interactions between cancer cells and engineered immune cells result in resistance to CAR therapies.
\end{abstract}

\section{Keywords}

Chimeric antigen receptor; resistance

\section{Introduction}

The first landmark clinical studies of chimeric antigen receptor (CAR)-engineered $\mathrm{T}$ cells in the treatment of hematologic malignancies were published less than a decade ago. ${ }^{1-3}$ Two of these studies report outcomes in three adult patients with relapsed or refractory chronic lymphocytic leukemia (CLL), a recalcitrant disease that is highly chemotherapy resistant,

\footnotetext{
*Corresponding Author: mej@pennmedicine.upenn.edu.

Conflicts of interest

N.S., E.O., Z.B., D.M.O. and J.J.M. are listed as investors on patents related to CAR T cell therapy and owned by the University of Pennsylvania and Novartis. J.J.M. is a consultant for Shanghai Unicar, Simcere of America, IASO Biotherapeutics, and Poseida Therapeutics, and receives research funding from Incyte, Novartis and the Parker Institute for Cancer Immunotherapy. E.O. is an employee of Novartis.

Publisher's Disclaimer: This is a PDF file of an unedited manuscript that has been accepted for publication. As a service to our customers we are providing this early version of the manuscript. The manuscript will undergo copyediting, typesetting, and review of the resulting proof before it is published in its final form. Please note that during the production process errors may be discovered which could affect the content, and all legal disclaimers that apply to the journal pertain.
} 
treated as part of a phase I study of CD19-targeted CAR T cells. All three patients experienced disease regression, and two achieved complete disease remission. These remarkable results paved the way for trials in pediatric patients with relapsed and refractory acute lymphoblastic leukemia (ALL), another highly challenging disease setting. Independent studies performed at multiple centers have demonstrated remarkably similar outcomes of CD19 CAR T cells in pediatric ALL, with complete remission rates consistently between 70-90\%. ${ }^{4-7}$ Investigation of CD19 CAR T cells has been expanded to non-Hodgkin lymphoma, where response rates have been more modest than those seen in ALL, however remain impressive. ${ }^{1,8,9}$ These results led to the FDA approval of two CD19 CAR T cell products in 2017: axicabtagene ciloleucel, approved for adults with relapsed or refractory non-Hodgkin lymphoma, and tisagenlecleucel, approved for the same indication in adults as well as in children with relapsed ALL. Integration of these new treatments into the clinical armamentarium has fundamentally changed the approach to patients with difficult-to-treat disease, and the flood of clinical trials evaluating new CAR-based products provides a glimpse into the future of this therapy and its role in modern management of hematologic malignancies.

We are now nine years removed from the initial reports of success using CAR T cells, and larger clinical trials have had time to mature. Close examination of the clinical trial data in lymphoma and ALL reveal important clues into the nature of CAR T cell success and failure. Outcomes in Non-Hodgkin's Lymphoma (NHL) and chronic lymphocytic leukemia (CLL) reveal that clinical failures are primarily characterized by an up-front lack of disease response. Patients that experience anti-tumor responses, however, appear to maintain those responses (Figure 1), with long-term remission occurring in $\sim 26-40 \%$ of patients treated. Intriguingly, outcomes in patients with ALL appear quite different. Long-term data confirm that $\sim 80 \%$ of patients will achieve complete remission, but that $\sim 40 \%$ of these patients will eventually relapse. Furthermore, targeted treatment of multiple myeloma with anti-B cell maturation antigen (BCMA)-specific CARs contributes to our experience in the field and provides additional insight into mechanisms of response and resistance to autologous, CARengineered $\mathrm{T}$ cell therapies. This difference in durability of response has many potential mechanisms, related to tumor-intrinsic and -extrinsic pathways to resistance.

In this review, we summarize the most recent clinical and pre-clinical work that has been done to identify the tumor-intrinsic and -extrinsic mechanistic bases of resistance to CAR T cell therapy. We focus on how interactions between tumor cells and $\mathrm{T}$ cells govern failure of these therapies, using models of both hematologic and solid malignancies. Finally, we highlight exciting areas of active research that may challenge traditional dogma about the fundamental mechanisms driving resistance.

\section{Tumor-intrinsic resistance}

Genetic mechanisms-A large proportion of patient with ALL treated with CD19 CAR $\mathrm{T}$ cells will relapse after achieving an initial complete remission. A seminal observation was that many of these relapsed leukemias demonstrated lack of surface CD19 expression (Figure 2). ${ }^{5,10} \mathrm{CD} 19$ status is usually assessed by flow cytometry of CD19 surface expression, ${ }^{11}$ categorizing patients as either CD19+ or CD19-. In CD19+ relapse surface 
CD19 is maintained and relapse is instead associated with lack of persistence of CAR T cells, often due to poor $\mathrm{T}$ cell function as discussed later in this review. In CD19- relapse, the absence of CD19 on the cell surface enables the tumor to evade CAR T-mediated recognition and clearance, despite CAR T cell persistence. Retreatment with an anti-CD19 therapy has not been successful for CD19- relapse, and patient outcomes are poor.

To date, there have been multiple reported mechanisms for CD19- relapse, including antigen escape via loss of function mutations accompanied by loss of heterozygosity (LOH) at the $C D 19$ locus, ${ }^{12}$ alternative splicing of $C D 19,{ }^{13}$ lineage switching, ${ }^{14,15}$ and CD19 masking. ${ }^{16}$ There have also been reports of antigen loss by dysregulated membrane trafficking of CD19 17 and reduction of target cell antigen concentration by trogocytosis. ${ }^{18}$

The combination of loss of function mutations coupled with $\mathrm{LOH}$ in $C D 19$ has been identified as a primary resistance mechanism for CD19- relapse in two phase II anti-CD19 CAR T cell clinical trials in children and young adults with B-ALL. ${ }^{12}$ A cohort of 12 patients with relapsed leukemia that entirely or almost entirely lacked surface CD19 expression was evaluated. DNA and/or RNA sequencing was performed on bone marrow and/or peripheral blood specimens collected at the time of screening and within 14 days of relapse from each of the 12 patients. DNA and RNA sequencing were used to identify short nucleotide variants (SNVs), as well as insertions or deletions across CD19 and other B cell related genes. DNA sequencing was further utilized for $\mathrm{LOH}$ and focal copy number alteration calling. At the time of relapse, leukemias from all patients were identified to harbor mutations in the CD19 extracellular or transmembrane (TM) coding regions that were not present at the time of screening. In addition to genetic mutations, $\mathrm{LOH}$ at $C D 19$ was observed in a majority of patients at relapse, suggesting a mechanism for biallelic gene silencing. The authors concluded that mutations were present in the vast majority of resistant ALL cells, and predictive modeling suggested that these specific mutations lead to either a truncated or non-functional TM domain, with consequent loss of cell surface CD19.

In a preceding report, Sotillo et al. performed DNA and RNA sequencing on relapse specimens from four CD19- relapsed leukemias. Loss of function mutations in CD19after disease relapse were reported in three of the patients and $\mathrm{LOH}$ was reported in two of these three patients. The one wild-type relapse was found in a later report to be resistant due to CD19 masking. ${ }^{16}$ The authors hypothesized that the frequency of mutations found in the three relapse patients alone could not fully explain the complete loss of surface CD19. RNA sequencing analysis suggested the existence of $C D 19$ isoforms with alterative splicing of exon 2, encoding the binding site of the anti-CD19 CAR, or exons 5 and 6, encoding the transmembrane domain. In vitro modeling demonstrated that increased alternative splicing of exon 2, 5 and 6 in $C D 19$ led to loss of surface CD19 expression. The authors hypothesized that exon 2 splicing eliminates the epitope binding site, and that employment of a CAR T targeting an alternative CD19 ectodomain (outside of exon 2) would be a possible approach for treating these patients. ${ }^{13}$

CD19- relapse has also been observed following other CD19-directed immunotherapies, suggesting that CD19 loss is not CAR-specific but driven by target-dependent selective pressure. ${ }^{19,20}$ It is unknown whether the mechanism of CD19 loss are the same in CAR T 
cell therapy and other CD19-targeting modalities. In one study, 5/17 patients treated in three phase 2 and one phase 3 clinical trials with blinatumomab, a CD19-directed monoclonal antibody, were found to have $C D 19$ mutations. 4/5 patients had tumor samples compatible with $\mathrm{LOH}$ and in $4 / 4$ patients $\mathrm{LOH}$ was present in $C D 19 .{ }^{21}$ The implication from this study is that some patients treated with blinatumomab experience CD19- relapse from CD19 mutations with $\mathrm{LOH}$, as seen with CAR T cell treated patients. However, other patients in this study did not appear to have mutations in $C D 19$. The authors suggested that this may be due to stronger selection pressure from CAR T cell therapy compared to blinatumomab, which also has a lower incidence of CD19- relapse. The 12/17 patients without mutations may lose CD19 protein expression by alternative splicing of $C D 19$, or other posttranscriptional mechanisms. In another study of a CD19- relapse following blinatumomab, disrupted CD19 membrane export was identified. Interestingly in this study, neither CD19 mutations nor alternative splicing of $C D 19$ at the time of relapse were identified. ${ }^{17}$ As CD19-targeting strategies expand, it will be important to integrate knowledge about resistance mechanisms to gain a more complete understanding of what drives CD19 loss in each treatment setting.

It remains unclear if the genetic mechanisms driving CD19- relapse exist in cancer cells a priori or are acquired following treatment. In Orlando et al. and Sotillo et al., CD19 mutational and transcriptional variants were not detected in the pre-treatment samples. ${ }^{12,13}$ However, B-ALL cells with $C D 19$ variants may exist prior to treatment at frequencies that are below the limit of detection of next generation sequencing. Treatment with CD19directed immunotherapy may allow selective survival of pre-existing CD19-negative clones. An alternative hypothesis is that the $C D 19$ variants are acquired post-treatment upon selective pressure of the therapy. MRD detection via deep $\operatorname{IgH}-\mathrm{V}(\mathrm{D}) \mathrm{J}$ next generation sequencing may now shed light on these questions. ${ }^{22}$

Finally, overcoming resistance driven by target antigen loss is not a unique challenge to CD19 targeted therapies but has been seen across a wide range of targeted therapies. For example, loss of $C D 20$ expression was seen after treatment with rituximab, a monoclonal anti-CD20 antibody. ${ }^{23}$

Much work is now being focused on methods to avert CD19- relapse. Since mutations have been reported throughout $C D 19$, retreatment or targeting an alternative CD19 ectodomain is unlikely to be successful. Significant effort is being dedicated to dual-antigen targeting as a means of evading antigen-negative relapse. In Orlando et al., all CD19- relapsed disease was CD22+ at screening and continued to have CD22+ cells present at relapse, suggesting that a combination CAR approach, such as anti-CD22 CAR T cells in combination with CAR19 therapy, may be effective.

Antigen density-Unlike peptide antigen-specific CD4+ or CD8+ T cell responses where mutations in the antigen processing and presentation machinery have frequently been observed,${ }^{24}$ those pathways do not play any role in the surface expression of CAR-targeted antigens. Instead, the mechanism of escape from CAR $\mathrm{T}$ cell detection likely reflects the functional avidity of the CAR. For instance, work from our laboratory (Zhao Y, Lundh, Maus, Melenhorst, June et al., unpublished observations) has revealed that minute quantities 
of CD19 protein are required to elicit lytic activity by CAR T-cells. Hence, only complete loss of antigen allows a CD19-expressing tumor to escape CAR T cell detection. ${ }^{4,5,10}$ Over the years both epigenetic ${ }^{14,15}$ and genetic ${ }^{12,13}$ mechanisms have been elucidated in this setting as outlined elsewhere in this paper.

Antigen escape in CAR T-cell therapies targeting the B cell antigen CD22, however, appear to be mechanistically distinct. Fry and colleagues recently reported their findings of antiCD22 CAR T cell therapy in B-ALL wherein 12/21 patients achieved complete remission; 9/12 experienced an MRD-negative CR. Eight of 12 patients, however, relapsed 1.5-12 months post-infusion. ${ }^{25}$ Intriguingly, all relapses were associated with reduced CD22 protein expression. Genomic analysis demonstrated that CD22 copy number profiles remained stable in the two patients studied, and none of the samples revealed the acquisition of mutations within the CD22 locus. RNA-sequencing analysis of samples prior to and following CAR T cell infusion did not identify a reduced CD22 expression level after treatment. Furthermore, whereas Sotillo and colleagues previously identified alternative splicing as a contributing factor to anti-CD19 CAR T cell escape ${ }^{13}$, Fry and colleagues observed no evidence of alternative CD22 isoforms underlying the reduced CD22 protein expression levels on tumor cells. These data therefore suggested that CD22 surface protein expression levels on tumor cells were regulated at the post-transcriptional level. A preclinical model consisting of a human ALL cell line reconstituted with low, intermediate, or high CD22 expression levels showed that mice infused with CD22 ${ }^{\text {low }}$ leukemia cells demonstrated negligible responses to CD22 CAR T cell therapy. Things findings suggest that the role of antigen density may vary depending on the target antigen, the CAR itself, or both.

CD20-specific CAR T cells also appear to require higher antigen expression levels for full activation. ${ }^{26}$ Beyond surface recognition of target antigens, binding of soluble antigen to the CAR may impede the ability of CAR T cells to eliminate target cells. A recent report brings this hypothesis into question for CAR T cells targeting the plasma cell protein $\mathrm{B}$ cell maturation antigen (BCMA). This protein is over-expressed by myeloma cells, and in some cases at several logs higher than on normal plasma cells in healthy individuals. BCMA is, however, also shed by plasma cells and can be used as a biomarker for myeloma tumor burden. Theoretically, high levels of soluble BCMA could interfere with CAR T cell function by coating CAR T cells and thereby function as an "antigen masking" mechanism. However, no evidence exists that this negatively impacts BCMA-targeted CAR T cells, ${ }^{27}$ and the growing number of clinical trials targeting BCMA will certainly shed more light on this evolving topic.

Antigen masking-The current method of manufacturing autologous T cell therapy products begins with collection of peripheral blood leukocytes via leukapheresis, followed by $\mathrm{T}$ cell enrichment. $\mathrm{T}$ cells are then activated using CD3/CD28 stimulatory beads, and the CAR transgene is delivered using a viral gene therapy vector (most often retrovirus or lentivirus). Engineered cells are then expanded in vitro and formulated/cryopreserved at the conclusion of manufacturing. The variation of starting material of patients comes from difference in composition of $\mathrm{T}$ lymphocytes (differences in memory subtypes, expression of exhaustion markers ${ }^{28,29}$ and number of regulatory $\mathrm{T}$ cells ${ }^{30}$ ) and non-T cell leukocytes. The 
presence of non-T cell leukocytes such as myeloid cells, monocytes and granulocytes from patients may dampen the $\mathrm{T}$ cell stimulation and expansion during the manufacturing ${ }^{31-35}$. In addition, circulating malignant cells may also serve as another inhibitor of $\mathrm{T}$ cell proliferation ${ }^{28}$. In the absence of fluorescence- or magnetic cell sorting, common densityand size-based methods are incapable of separating $\mathrm{T}$ cell subsets from non-T leukocytes prior to genetic engineering. Thus, it is still plausible that non-T cell leukocytes present in the apheresis product could undergo genetic engineering during CAR T cell manufacturing. Despite these variations in the starting material, multiple groups have demonstrated that final manufactured products are regularly $>80 \% \mathrm{~T}$ cells, despite variations in manufacturing protocols themselves ${ }^{36-38}$.

Our group recently reported the unintentional transduction of a single leukemia cell with the anti-CD19 CAR transgene, and re-infusion of CAR19 T cells along with CAR19-modified tumor cells in a patient with B-cell ALL ${ }^{16}$. This case was brought to light after the patient was identified to have relapsed with a seemingly CD19-negative leukemia. Molecular analysis revealed that these leukemic cells did not harbor mutations in $C D 19$ or the coreceptor $C D 81$, necessary for CD19 membrane delivery, or demonstrate evidence of alternative $C D 19$ splicing. Intriguingly, while CD19 CAR T cells underwent contraction in the peripheral blood, qPCR analysis revealed persistence of the CAR transgene. Further investigation demonstrated that the relapsed ALL had been engineered with two copies of the CD19 CAR transgene, which resulted in the persist expression of the CAR in cis with CD19 on the leukemic cell surface. Binding of the CAR to CD19 on the same cell resulted in masking of the CAR epitope on CD19, preventing detection by CD19-targeted CAR T cells, and allowing escape of a seemingly antigen-negative leukemic clone. This finding, although the only such identified case thus far, highlights the urgent need for increasing the efficiency of $\mathrm{T}$ cell enrichment prior to genetic transduction during manufacture, specifically as clinical CAR T cell manufacturing is broadened to more centers. This also underscores the need for the development of consistent manufacturing processes that will prevent such cases in the future.

Tumor microenvironment-The tumor microenvironment (TME) encompasses both non-cancerous cells and non-cellular tissue. In brain tumors, these components include myeloid cells, with a macrophage predominance, endothelial cells, pericytes, and fibroblasts. ${ }^{39}$ Little is known regarding the response of the TME to immunotherapies, other than that it plays a significant role in tumor response. ${ }^{40}$ There have been very few neoadjuvant clinical trials that allow for surgical sampling of tumor tissue and in-depth analysis of the TME after immunotherapy, including immune checkpoint blockade, peptide vaccines or cell therapies. Additionally, relatively few CAR T cell clinical trials have evaluated TME composition after treatment. In glioblastoma (GBM), baseline immunosuppression within the TME has been demonstrated in several studies. ${ }^{41,42}$ Systemic delivery of Epidermal Growth Factor Receptor variant III (EGFRvIII)-targeted CAR T cells in patients with refractory GBM has demonstrated significant limitations in T cell entry, in situ proliferation and activation, as well as EGFRvIII antigen loss. ${ }^{40}$ Evaluation of primary patient tissue collected 7-14 days after CAR $\mathrm{T}$ cell infusion revealed that functional CAR $\mathrm{T}$ activation was accompanied by a robust immunosuppressive response that was felt to limit efficacy of CAR T cell infusion. 
The GBM TME showed induction of PD-1, PDL-1 and IDO- 1 along with a large FoxP3 ${ }^{+} \mathrm{T}$ cell population, suggesting significant upregulation of immunosuppressive surface proteins as well as regulatory $\mathrm{T}$ cell infiltration. An ongoing Phase I trial targeting ROR1-expressing non-small cell lung cancers and triple negative breast cancers found upregulation of immunosuppressive receptors on the CAR T cells at the time of peak expansion. ${ }^{43}$

Conversely, CEA-targeted CAR T cells isolated from liver metastases of $\mathrm{CEA}^{+}$tumors demonstrated a decrease in PD-L1 and IDO. ${ }^{44}$ The CAR T cells were administered directly to the hepatic blood supply and the resultant CAR $\mathrm{T}$ activity promoted a regional proinflammatory milieu in the vicinity of the tumors. Collectively, these observations suggest that the microenvironment may play distinct roles depending on tumor histology.

A significant limitation in modeling of CAR T cell efficacy is preclinical evaluation in immunodeficient NOD-scid-IL-2R $\gamma c^{\text {null }}$ (NSG) mice. ${ }^{45}$ Interactions between the TME, tumor, and CAR T cells are limited by the lack of endogenous immune system. Despite these limitations, important observations regarding immune cell interactions can be made using the NSG system. Several in vivo studies in NSG mice have shown immune checkpoint upregulation upon tumor challenge with CAR T cells. CAR T cells targeting EGFRvIII or IL13Ra2 demonstrated increased expression of immunosuppressive surface markers PD1, TIM3 and CTLA4 after co-culture with patient-derived xenograft D270 glioma models. ${ }^{46}$ This in vitro assessment was corroborated by increased survival in D270-bearing animals when CAR T cells were combined with distinct immune checkpoint blockade. In a complementary study, hepatocellular carcinoma cells (PLC/PRF/5) exhibited increased PDL1 expression when treated with GPC3-targeting CAR T cells. ${ }^{47}$

Several important points emerge from these early observations. First, acquisition of tissue post-CAR T treatment will continue to provide critical insight into comprehensive TME responses to adoptive cell therapy. This may require prioritizing different clinical trial designs, with an emphasis on surgical resection and TME evaluation after CAR delivery. This approach has already provided valuable insight into tumor infiltrating lymphocyte composition after peptide vaccine therapy ${ }^{48}$ and checkpoint blockade therapy ${ }^{49}$ Second, if additional studies confirm that the response of the TME in solid cancers is immunosuppressive and antagonistic, additional hurdles to successful treatment will need to be defined. Lastly, technologies now exist to explore the TME in great depth and at the single-cell level to more articulately define the cellular architecture of the TME pre- and post-adoptive cell therapy. Rational design of future immuno-oncology combinations that may be more likely to result in clinical efficacy should follow.

\section{T cell failure}

CAR immunogenicity-Persistence of infused CAR T cells is an important correlate of anti-tumor efficacy. Until recently, most CARs evaluated clinically have derived their single chain variable fragment ( $\mathrm{scFv}$ ) from non-human species, and mounting evidence suggests that this may play a direct role in limiting persistence of adoptively transferred cells. Kershaw et al. ${ }^{50}$ targeted the alpha-folate receptor, overexpressed on ovarian cancer cells, using a first-generation $\mathrm{CD} 3 \zeta \mathrm{CAR}$. The $\mathrm{scFv}$ was based on the murine monoclonal antibody (mAb) MOv18. In this phase I trial, three of the tested six cases developed an 
inhibitory serum factor which blocked the anti-tumor response (Figure 3). Interestingly, protein $\mathrm{G}$ pre-incubation in the post-treatment serum completely removed this inhibitory effect, suggesting that anti-CAR antibodies were responsible for this inhibition. Similarly, Lamers et al. ${ }^{51,52}$ targeting renal cell carcinoma with an anti-carbonic anhydrase-IX (CAIX) first-generation murine scFv (mAb G250)-based CAR observed persistence for a few weeks. They demonstrated that most patients developed anti-CAR B cell and T cell responses. Similar observations were made targeting CD19 and CD20 CARs. ${ }^{53}$ Though these studies revealed an adaptive immune response after CAR T cell infusion, these data were correlative in nature. Furthermore, the limited persistence observed of the infused cells could also be explained by the design of the CAR, which lacked a co-stimulatory domain. More recently 17 patients with multiple myeloma were treated with a 4-1BB/CD3 $\zeta$-signaling CAR containing two B cell maturation antigen (BCMA)-specific, camelid-derived antibody fragments. ${ }^{54}$ The data indicated that among seven patients harboring a high-level of antiCAR antibody response, six cases relapsed or progressed 6 months after infusion. This incidence was significantly higher than that in patients without detected humoral immunogenicity. In patients with a high proportion of anti-CAR antibody, the CAR-T levels were very low. A similar clinical correlation between reduced clinical efficacy and CAR immunogenicity were made by Turtle et al., who demonstrated that the re-infusion of lymphoma and ALL patients with the original second-generation CAR did not result in their expansion and tumor reduction. ${ }^{9,55}$ These authors were able to map the anti-CAR T cell response to a specific domain in the murine FMC63-based CAR, offering the opportunity of de-immunizing this CAR for enhanced clinical efficacy. ${ }^{56}$

Several groups have used cytoreducing agents to enhance engraftment and prolong the tumor-targeting effect of CAR T cells. Indeed, the addition of fludarabine to the lymphodepletion regimen strongly enhanced the CAR-T cells proliferation and improved response duration. ${ }^{9,55,57}$ One trial in multiple myeloma found that among seven patients treated with cyclophosphamide plus fludarabine, only one patient had an anti-CAR immune response, while in 8 evaluated patients receiving single agent cyclophosphamide, 6 had detectable anti-CAR responses. This evidence further indicated that fludarabine may reduce, inhibit, or delay anti-CAR antibody production. ${ }^{54}$ However, the underlying mechanism of fludarabine in reduced immunogenicity deserves further investigation.

Since the possibility of reduced anti-tumor activity of a non-human antibody-based CAR looms large over the field, researchers have begun to develop fully human CAR to overcome the issue of transgene immunogenicity. ${ }^{58,59} \mathrm{~A}$ fully human scFv-based anti-CD19 CAR (JCAR021) has been initially investigated in 7 patients with ALL or NHL enrolled in a phase I trial (NCT03103971). The clinical toxicity was favorable and 4 out of 7 patients achieved a partial or complete response. Importantly, JCAR021 persisted in peripheral blood for $>4$ months post infusion in all patients. ${ }^{60}$ Other groups have also transitioned to using humanized or fully human CAR T cells targeting BCMA ${ }^{61,62}$ or mesothelin (NCT03054298 and NCT03323944). Although it remains pre-mature to draw conclusions based on these early trials, trends suggest that use of human scFvs may enhance CAR T cell function.

T cell product dysfunction-Unlike conventional therapies, CAR T cells and other cellbased therapeutics are influenced by conditions that affect cellular fitness, such as metabolic 
stress, the presence of immune-suppressive cytokines and cell-to-cell interactions. In addition, intrinsic cell fitness must be considered, as CAR T cells made from "low quality" cells have been shown not to perform as well as CAR T cells made from "high quality" cells. ${ }^{63,64}$ The effect of $\mathrm{T}$ cell quality on CAR T cell therapy is best underscored by comparing the differences in response between CART19-treated ALL patients and CLL patients. While many factors differentiate ALL from CLL, the same CART19 products that yield $>80 \%$ complete remissions in pediatric ALL result in a response rate of only $26 \%$ in adults with CLL, highlighting a stark distinction in efficacy between these two cell therapy products. ${ }^{65}$ Beyond fundamental differences in cancer cell biology, it has been shown that cancer type has a direct influence on CAR T cell fitness. ${ }^{64,66}$

Several factors likely contribute to the development of T cell dysfunction in CLL. First, CLL patients have a median age of diagnosis of 70 years, making their $\mathrm{T}$ cells chronologically older and less fit as a result of a waning immune system and a higher proportion of terminally differentiated effector memory cells. ${ }^{67}$ These cells inherently have limited immune responsiveness and secrete cytokines such as IFN $\gamma$ and IL-4 that support the growth of the tumor cells and have been shown to induce the expression of anti-apoptotic proteins such as BCL-2 in CLL cells themselves. ${ }^{68,69}$ These cells also demonstrate signs of functional impairments beyond simple aging; previous work has shown that chronic lymphoid malignancies directly deplete the circulating naïve T cell pool. ${ }^{64}$ Further highlighting the importance of $\mathrm{T}$ cell quality as defined by memory state, it is possible to predict which CLL patients will respond based on the frequency of naïve or early memory cells present prior to CAR T cell manufacturing; patients with a higher proportion of naïve or early memory CD8+ T cells have a greater response rate than patients with few or no such T cells. ${ }^{63}$

In addition to skewing of memory populations, T cells from CLL patients demonstrate impaired cytotoxic function, impaired immune synapse formation, low-level, dysregulated cytokine production, and prolonged expression of inhibitory receptors. ${ }^{28,70}$ This dysfunctional state shares similarities to the classical $\mathrm{T}$ cell exhaustion seen in chronic infection models. ${ }^{70}$ Actin polymerization and polarization are critical for the formation of the immune synapse, and exposure to CLL has been shown to be sufficient to impair synapse formation in even healthy donor T cells. ${ }^{71,72}$ This co-culture alters Rho GTPase signaling in T cells by upregulating Cdc 42 and downregulating Rac1 and RhoA. Cdc 42 is a negative regulator and Rac1/RhoA are positive regulators of the integrin LFA-1, a critical component of the immune synapse. In concert, this results in significantly decreased adhesive function of LFA-1, preventing effective synapse formation. ${ }^{73}$

Altered cytokine production of T cells from CLL patients contributes to tumor escape in several ways. First, while CLL T cells do produce a variety of effector cytokines, the levels of production are significantly lower than those produced by healthy T cells. ${ }^{28,63}$ This may be due to decreased cytokine production or direct uptake of IL-2 by CLL cells themselves. Second, CLL cells are known to exert immunosuppressive cytokine activity, secreting TGF $\beta$ and serving as an IL-2 sink. ${ }^{74,75}$ This IL-2 sinking further impairs immune responses, as IL-2 is required for full T cell activation. Furthermore, CLL cells express and release high level of the inhibitory molecule CD200. ${ }^{76}$ Tumor cells from poor prognosis CLL patients 
expressed significantly higher levels of this inhibitory molecule. ${ }^{77}$ Additionally, CD200 blocking stabilized T cell-CLL cell interactions, suggesting that the CD200-CD200R axis contributes to reduced $\mathrm{T}$ cell reactivity against CLL and possibly other tumors as well. Finally, T cells from CLL patients have high, sustained expression of inhibitory receptors including PD-1, TIM3, and LAG3. Inhibitory receptor expression can both destabilize the immune synapse and suppress functional immune responses. ${ }^{67,78,79}$

Abortive CAR T cell activation-During a normal immune response, co-stimulatory molecule expression on antigen presenting cells is a requirement for inducing a productive $\mathrm{T}$ cell response. When the TCR is engaged in the absence of co-stimulation, T cells become anergic and fail to differentiate into mature cytotoxic T lymphocytes. ${ }^{80} \mathrm{CAR} \mathrm{T}$ cells are unique in that the chimeric receptor contains co-stimulatory intracellular signaling domains. Currently, there is no evidence that CAR T cells require additional co-stimulation from tumor cells. However, co-stimulatory signaling molecule expression varies by disease type. ALL blasts express high levels of CD86, CD40, CD27, and CD70, whereas CLL cells express low levels of CD80 and CD86, and high levels of CD40. ${ }^{81,82}$ Interestingly, CLL cells can promote the down-regulation of CD40 ligand on CD4 T cells. Activated T cells upregulate CD40, which serves as another important costimulatory factor, and its loss may further blunt $\mathrm{T}$ cell responses in this disease. In addition, constitutive CD40 ligand expression on CAR T cells has been shown to enhance their tumor-targeting capability. ${ }^{83}$ Further work is necessary to determine if the differences in co-stimulatory molecule expression between different tumor types contribute to the poor responses seen in CLL, and potentially other diseases. We may predict that if CAR T cells are deprived of necessary costimulatory signals not present on their targets, these cells may become anergic similar to their native counterparts, impairing anti-tumor function.

\section{Conclusions and Future Directions}

CAR-based $\mathrm{T}$ cell immunotherapy represents a paradigm-shift in the therapeutic approach to patients with cancer. It was recognized as the "Advance of the Year" by the American Society of Clinical Oncology in 2018, highlighting its significance in the field of oncology. Now that the "honeymoon" has ended, however, it is our responsibility to reflect on the current status of this therapy and recognize its limitations.

CAR T cell product manufacturing remains a highly-specialized, labor-intensive and individualized practice. While many centers have become certified to deliver this therapy, it is far from broadly available across the US, not to mention less-developed countries. Beyond the barriers to accessibility, successful manufacturing of a clinically-viable product remains a significant hurdle for many patients. ${ }^{64}$ While these therapies represent an exciting new addition to the oncologist's armamentarium, how they integrate with current therapies, specifically hematopoietic cell transplantation, remains unresolved. Finally, the high cost also places a new strain on the healthcare system. A recent study has attempted to evaluate cost-effectiveness of this therapy, with mixed conclusions. ${ }^{84}$

Nestled within these other obstacles is the clinical and scientific hurdle of therapeutic failure. The traditional approach to failure has categorized it into two forms: lack of response 
or emergence of CD19+ relapse attributed to T cell failure, and CAR immunogenicity, or adaptive resistance and CD19- relapse attributed to tumor evasion (Figure 1). As outlined in this review, several mechanisms have been identified as leading to each of these outcomes. While it has been instructive to consider these as distinct biological entities leading to therapeutic failure, the reality is likely not so simple. Our group has recently identified that tumor-intrinsic qualities can lead to resistance to CAR T cell cytotoxicity, and that this resistance in turn leads to the development of CAR $\mathrm{T}$ cell dysfunction and failure as a result of persistent antigen stimulation. ${ }^{85}$ This mechanism highlights the dynamic interplay between $T$ cells and tumor cells that can lead to therapeutic failure, and offers a new model for resistance to CAR T cell therapy.

A great deal of effort has now been dedicated to surmounting resistance. Several groups have developed strategies to target two antigens on the tumor cell surface in an effort to avert antigen-negative escape. ${ }^{25,86-88}$ Targeting of the TME has already shown benefit in the efficacy of CAR therapies in solid tumors, ${ }^{89}$ highlighting a pathway to overcome some of the hurdles imposed by immunosuppressive non-cancerous tissue. Finally, combination therapies are emerging as an exciting approach to overcome limitations in CAR T cell efficacy and toxicity. Thus far these have included ibrutinib, ${ }^{29}$ dasatinib ${ }^{90}$ and checkpoint inhibitor antagonists; ${ }^{91,92}$ surely this list will continue to grow as novel combinations are evaluated pre-clinical and in patients.

Resistance to CAR T cells is a primary barrier to its broader efficacy. Rapidly evolving research into the mechanisms responsible, as well as strategies to overcome resistance, provide great promise to patients in need of these innovative therapies.

\section{Acknowledgments}

Funding

This work was supported by Novartis Institute for Biomedical Research (ND, EO, JunX, ZB, DOR, JJM), RO1CA241762 (JJM), P01CA214278 (JJM) and the Parker Institute for Cancer Immunotherapy (MAC, JJM). Dr. Jie Xu is supported by a fellowship grant from the Ruijin Hospital affiliated with Shanghai Jiao Tong University School of Medicine, Shanghai, PR China.

\section{References}

1. Kochenderfer JN et al. Eradication of B-lineage cells and regression of lymphoma in a patient treated with autologous T cells genetically engineered to recognize CD19. Blood 116, 4099-4102, doi:10.1182/blood-2010-04-281931 (2010). [PubMed: 20668228]

2. Porter DL, Levine BL, Kalos M, Bagg A. \& June CH Chimeric antigen receptor-modified T cells in chronic lymphoid leukemia. N Engl J Med 365, 725-733, doi:10.1056/NEJMoa1103849 (2011). [PubMed: 21830940]

3. Kalos M. et al. T cells with chimeric antigen receptors have potent antitumor effects and can establish memory in patients with advanced leukemia. Sci Transl Med 3, 95ra73, doi:10.1126/ scitranslmed.3002842 (2011).

4. Maude SL et al. Chimeric antigen receptor T cells for sustained remissions in leukemia. N Engl J Med 371, 1507-1517, doi:10.1056/NEJMoa1407222 (2014). [PubMed: 25317870]

5. Maude SL et al. Tisagenlecleucel in Children and Young Adults with B-Cell Lymphoblastic Leukemia. N Engl J Med 378, 439-448, doi:10.1056/NEJMoa1709866 (2018). [PubMed: 29385370] 
6. Lee DW et al. T cells expressing CD19 chimeric antigen receptors for acute lymphoblastic leukaemia in children and young adults: a phase 1 dose-escalation trial. Lancet $385,517-528$, doi:10.1016/S0140-6736(14)61403-3 (2015). [PubMed: 25319501]

7. Gardner RA et al. Intent-to-treat leukemia remission by CD19 CAR T cells of defined formulation and dose in children and young adults. Blood 129, 3322-3331, doi:10.1182/blood-2017-02-769208 (2017). [PubMed: 28408462]

8. Schuster SJ et al. Tisagenlecleucel in Adult Relapsed or Refractory Diffuse Large B-Cell Lymphoma. N Engl J Med 380, 45-56, doi:10.1056/NEJMoa1804980 (2019). [PubMed: 30501490]

9. Turtle CJ et al. Immunotherapy of non-Hodgkin's lymphoma with a defined ratio of CD8+ and CD4+ CD19-specific chimeric antigen receptor-modified T cells. Sci Transl Med 8, 355ra116, doi:10.1126/scitranslmed.aaf8621 (2016).

10. Grupp SA et al. Chimeric antigen receptor-modified T cells for acute lymphoid leukemia. N Engl J Med 368, 1509-1518, doi:10.1056/NEJMoa1215134 (2013). [PubMed: 23527958]

11. Chiaretti S, Zini G. \& Bassan R. Diagnosis and subclassification of acute lymphoblastic leukemia. Mediterr J Hematol Infect Dis 6, e2014073, doi:10.4084/MJHID.2014.073 (2014).

12. Orlando EJ et al. Genetic mechanisms of target antigen loss in CAR19 therapy of acute lymphoblastic leukemia. Nat Med 24, 1504-1506, doi:10.1038/s41591-018-0146-z (2018). [PubMed: 30275569]

13. Sotillo E. et al. Convergence of Acquired Mutations and Alternative Splicing of CD19 Enables Resistance to CART-19 Immunotherapy. Cancer Discov 5, 1282-1295, doi:10.1158/2159-8290.CD-15-1020 (2015). [PubMed: 26516065]

14. Jacoby E. et al. CD19 CAR immune pressure induces B-precursor acute lymphoblastic leukaemia lineage switch exposing inherent leukaemic plasticity. Nat Commun 7, 12320, doi:10.1038/ ncomms 12320 (2016).

15. Gardner R. et al. Acquisition of a CD19-negative myeloid phenotype allows immune escape of MLL-rearranged B-ALL from CD19 CAR-T-cell therapy. Blood 127, 24062410, doi:10.1182/ blood-2015-08-665547 (2016).

16. Ruella $\mathrm{M}$. et al. Induction of resistance to chimeric antigen receptor $\mathrm{T}$ cell therapy by transduction of a single leukemic B cell. Nat Med 24, 1499-1503, doi:10.1038/s41591-018-0201-9 (2018). [PubMed: 30275568]

17. Braig F. et al. Resistance to anti-CD19/CD3 BiTE in acute lymphoblastic leukemia may be mediated by disrupted CD19 membrane trafficking. Blood 129, 100-104, doi:10.1182/ blood-2016-05-718395 (2017). [PubMed: 27784674]

18. Hamieh M. et al. CAR T cell trogocytosis and cooperative killing regulate tumour antigen escape. Nature 568, 112-116, doi:10.1038/s41586-019-1054-1 (2019). [PubMed: 30918399]

19. Topp MS et al. Phase II trial of the anti-CD19 bispecific T cell-engager blinatumomab shows hematologic and molecular remissions in patients with relapsed or refractory B-precursor acute lymphoblastic leukemia. J Clin Oncol 32, 4134-4140, doi:10.1200/JCO.2014.56.3247 (2014). [PubMed: 25385737]

20. Zugmaier G. et al. Long-term survival and T-cell kinetics in relapsed/refractory ALL patients who achieved MRD response after blinatumomab treatment. Blood 126, 2578-2584, doi:10.1182/ blood-2015-06-649111 (2015). [PubMed: 26480933]

21. Kotrova M. e. a. CD19 MUTATION FREQUENCY AFTER BLINATUMOMAB TREATMENT IS LOWER THAN AFTER CAR T-CELLS TREATMENT. EHA Learning Center (2019).

22. Pulsipher MA et al. IgH-V(D)J NGS-MRD measurement pre- and early post-allotransplant defines very low- and very high-risk ALL patients. Blood 125, 3501-3508, doi:10.1182/ blood-2014-12-615757 (2015). [PubMed: 25862561]

23. Duman BB, Sahin B, Ergin M. \& Guvenc B. Loss of CD20 antigen expression after rituximab therapy of CD20 positive B cell lymphoma (diffuse large B cell extranodal marginal zone lymphoma combination): a case report and review of the literature. Med Oncol 29, 1223-1226, doi:10.1007/s12032-011-9955-3 (2012). [PubMed: 21805377]

24. Sharma P, Hu-Lieskovan S, Wargo JA \& Ribas A. Primary, Adaptive, and Acquired Resistance to Cancer Immunotherapy. Cell 168, 707-723, doi:10.1016/j.cell.2017.01.017 (2017). [PubMed: 28187290] 
25. Fry TJ et al. CD22-targeted CAR T cells induce remission in B-ALL that is naive or resistant to CD19-targeted CAR immunotherapy. Nat Med 24, 20-28, doi:10.1038/nm.4441 (2018). [PubMed: 29155426]

26. Watanabe K. et al. Target antigen density governs the efficacy of anti-CD20-CD28-CD3 zeta chimeric antigen receptor-modified effector CD8+ T cells. J Immunol 194, 911-920, doi:10.4049/ jimmunol.1402346 (2015). [PubMed: 25520398]

27. Cohen AD et al. B cell maturation antigen-specific CAR T cells are clinically active in multiple myeloma. J Clin Invest 129, 2210-2221, doi:10.1172/JCI126397 (2019). [PubMed: 30896447]

28. Riches JC et al. T cells from CLL patients exhibit features of T-cell exhaustion but retain capacity for cytokine production. Blood 121, 1612-1621, doi:10.1182/blood-2012-09-457531 (2013). [PubMed: 23247726]

29. Fraietta JA et al. Ibrutinib enhances chimeric antigen receptor T-cell engraftment and efficacy in leukemia. Blood 127, 1117-1127, doi:10.1182/blood-2015-11-679134 (2016). [PubMed: 26813675]

30. Woo EY et al. Cutting edge: Regulatory T cells from lung cancer patients directly inhibit autologous T cell proliferation. J Immunol 168, 4272-4276, doi:10.4049/jimmunol.168.9.4272 (2002). [PubMed: 11970966]

31. Almand B. et al. Increased production of immature myeloid cells in cancer patients: a mechanism of immunosuppression in cancer. J Immunol 166, 678-689, doi:10.4049/jimmunol.166.1.678 (2001). [PubMed: 11123353]

32. Filipazzi P. et al. Identification of a new subset of myeloid suppressor cells in peripheral blood of melanoma patients with modulation by a granulocyte-macrophage colony-stimulation factor-based antitumor vaccine. J Clin Oncol 25, 2546-2553, doi:10.1200/JCO.2006.08.5829 (2007). [PubMed: 17577033]

33. Stroncek DF et al. Myeloid cells in peripheral blood mononuclear cell concentrates inhibit the expansion of chimeric antigen receptor T cells. Cytotherapy 18, 893-901, doi:10.1016/ j.jcyt.2016.04.003 (2016). [PubMed: 27210719]

34. Stroncek DF et al. Elutriated lymphocytes for manufacturing chimeric antigen receptor T cells. J Transl Med 15, 59, doi:10.1186/s12967-017-1160-5 (2017). [PubMed: 28298232]

35. Pillay J, Tak T, Kamp VM \& Koenderman L. Immune suppression by neutrophils and granulocytic myeloid-derived suppressor cells: similarities and differences. Cell Mol Life Sci 70, 3813-3827, doi:10.1007/s00018-013-1286-4 (2013). [PubMed: 23423530]

36. Brentjens RJ et al. Safety and persistence of adoptively transferred autologous CD19-targeted T cells in patients with relapsed or chemotherapy refractory B-cell leukemias. Blood 118, 48174828, doi:10.1182/blood-2011-04-348540 (2011). [PubMed: 21849486]

37. Singh H, Moyes JS, Huls MH \& Cooper LJ Manufacture of T cells using the Sleeping Beauty system to enforce expression of a CD19-specific chimeric antigen receptor. Cancer Gene Ther 22, 95-100, doi:10.1038/cgt.2014.69 (2015). [PubMed: 25591810]

38. Tumaini B. et al. Simplified process for the production of anti-CD19-CAR-engineered T cells. Cytotherapy 15, 1406-1415, doi:10.1016/j.jcyt.2013.06.003 (2013). [PubMed: 23992830]

39. Quail DF \& Joyce JA The Microenvironmental Landscape of Brain Tumors. Cancer Cell 31, 326341, doi:10.1016/j.ccell.2017.02.009 (2017). [PubMed: 28292436]

40. O'Rourke DM et al. A single dose of peripherally infused EGFRvIII-directed CAR T cells mediates antigen loss and induces adaptive resistance in patients with recurrent glioblastoma. Sci Transl Med 9, doi:10.1126/scitranslmed.aaa0984 (2017).

41. Jackson C, Ruzevick J, Phallen J, Belcaid Z. \& Lim M. Challenges in immunotherapy presented by the glioblastoma multiforme microenvironment. Clin Dev Immunol 2011, 732413, doi:10.1155/2011/732413 (2011).

42. Razavi SM et al. Immune Evasion Strategies of Glioblastoma. Front Surg 3, 11, doi:10.3389/ fsurg.2016.00011 (2016). [PubMed: 26973839]

43. Specht JM et al. Abstract CT131: A phase I study of adoptive immunotherapy for advanced ROR1+ malignancies with defined subsets of autologous T cells expressing a ROR1-specific chimeric antigen receptor (ROR1-CAR). Cancer Research 78, CT131-CT131, doi:10.1158/1538-7445.am2018-ct131 (2018). 
44. Katz SC et al. HITM-SIR: phase Ib trial of intraarterial chimeric antigen receptor T-cell therapy and selective internal radiation therapy for $\mathrm{CEA}(+)$ liver metastases. Cancer Gene Ther, doi:10.1038/s41417-019-0104-z (2019).

45. Ishikawa F. et al. Development of functional human blood and immune systems in NOD/SCID/IL2 receptor \{gamma\} chain(null) mice. Blood 106, 1565-1573, doi:10.1182/blood-2005-02-0516 (2005). [PubMed: 15920010]

46. Yin Y. et al. Checkpoint Blockade Reverses Anergy in IL-13Ralpha2 Humanized scFv-Based CAR T Cells to Treat Murine and Canine Gliomas. Mol Ther Oncolytics 11, 20-38, doi:10.1016/ j.omto.2018.08.002 (2018). [PubMed: 30306125]

47. Guo X. et al. Disruption of PD-1 Enhanced the Anti-tumor Activity of Chimeric Antigen Receptor T Cells Against Hepatocellular Carcinoma. Front Pharmacol 9, 1118, doi:10.3389/ fphar.2018.01118 (2018). [PubMed: 30327605]

48. Keskin DB et al. Neoantigen vaccine generates intratumoral $\mathrm{T}$ cell responses in phase $\mathrm{Ib}$ glioblastoma trial. Nature 565, 234-239, doi:10.1038/s41586-018-0792-9 (2019). [PubMed: 30568305]

49. Cloughesy TF et al. Neoadjuvant anti-PD-1 immunotherapy promotes a survival benefit with intratumoral and systemic immune responses in recurrent glioblastoma. Nat Med 25, 477-486, doi:10.1038/s41591-018-0337-7 (2019). [PubMed: 30742122]

50. Kershaw $\mathrm{MH}$ et al. A phase I study on adoptive immunotherapy using gene-modified T cells for ovarian cancer. Clin Cancer Res 12, 6106-6115, doi:10.1158/1078-0432.CCR-06-1183 (2006). [PubMed: 17062687]

51. Lamers $\mathrm{CH}$ et al. Treatment of metastatic renal cell carcinoma with autologous T-lymphocytes genetically retargeted against carbonic anhydrase IX: first clinical experience. J Clin Oncol 24, e20-22, doi:10.1200/JCO.2006.05.9964 (2006). [PubMed: 16648493]

52. Lamers $\mathrm{CH}$ et al. Immune responses to transgene and retroviral vector in patients treated with ex vivo-engineered T cells. Blood 117, 72-82, doi:10.1182/blood-2010-07-294520 (2011). [PubMed: 20889925]

53. Jensen $\mathrm{MC}$ et al. Antitransgene rejection responses contribute to attenuated persistence of adoptively transferred CD20/CD19-specific chimeric antigen receptor redirected T cells in humans. Biol Blood Marrow Transplant 16, 1245-1256, doi:10.1016/j.bbmt.2010.03.014 (2010). [PubMed: 20304086]

54. Xu J. et al. Exploratory trial of a biepitopic CAR T-targeting B cell maturation antigen in relapsed/ refractory multiple myeloma. Proc Natl Acad Sci U S A 116, 9543-9551, doi:10.1073/ pnas.1819745116 (2019). [PubMed: 30988175]

55. Turtle CJ et al. CD19 CAR-T cells of defined CD4+:CD8+ composition in adult B cell ALL patients. J Clin Invest 126, 2123-2138, doi:10.1172/JCI85309 (2016). [PubMed: 27111235]

56. Cantor JR et al. Therapeutic enzyme deimmunization by combinatorial T-cell epitope removal using neutral drift. Proc Natl Acad Sci U S A 108, 1272-1277, doi:10.1073/pnas.1014739108 (2011). [PubMed: 21209329]

57. Haas AR et al. Phase I Study of Lentiviral-Transduced Chimeric Antigen Receptor-Modified T Cells Recognizing Mesothelin in Advanced Solid Cancers. Mol Ther, doi:10.1016/ j.ymthe.2019.07.015 (2019).

58. Sommermeyer D. et al. Fully human CD19-specific chimeric antigen receptors for T-cell therapy. Leukemia 31, 2191-2199, doi:10.1038/leu.2017.57 (2017). [PubMed: 28202953]

59. Mirzaei HR et al. Construction and functional characterization of a fully human anti-CD19 chimeric antigen receptor (huCAR)-expressing primary human T cells. J Cell Physiol 234, 92079215, doi:10.1002/jcp.27599 (2019). [PubMed: 30362586]

60. Gauthier J. e. a. Immunotherapy with T-Cells Engineered with a Chimeric Antigen Receptor Bearing a Human CD19-Binding Single Chain Variable Fragment for Relapsed or Refractory Acute Lymphoblastic Leukemia and B-Cell Non-Hodgkin Lymphoma. Blood 132, 1415 (2018).

61. Gregory T. e. a. Efficacy and Safety of P-Bcma-101 CAR-T Cells in Patients with Relapsed/ Refractory (r/r) Multiple Myeloma (MM). Blood 132, 1012 (2018). 
62. Mailankody S. JCARH125, Anti-BCMA CAR T-cell Therapy for Relapsed/Refractory Multiple Myeloma: Initial Proof of Concept Results from a Phase 1/2 Multicenter Study (EVOLVE). Blood 132, 957 (2018).

63. Fraietta JA et al. Determinants of response and resistance to CD19 chimeric antigen receptor (CAR) T cell therapy of chronic lymphocytic leukemia. Nat Med 24, 563-571, doi:10.1038/ s41591-018-0010-1 (2018). [PubMed: 29713085]

64. Singh N, Perazzelli J, Grupp SA \& Barrett DM Early memory phenotypes drive T cell proliferation in patients with pediatric malignancies. Sci Transl Med 8, 320ra323, doi:10.1126/ scitranslmed.aad5222 (2016).

65. Sallan SE Myths and lessons from the adult/pediatric interface in acute lymphoblastic leukemia. Hematology Am Soc Hematol Educ Program, 128-132, doi:10.1182/asheducation-2006.1.128 (2006). [PubMed: 17124051]

66. Das RK, Vernau L, Grupp SA \& Barrett DM Naive T-cell Deficits at Diagnosis and after Chemotherapy Impair Cell Therapy Potential in Pediatric Cancers. Cancer Discov 9, 492-499, doi:10.1158/2159-8290.CD-18-1314 (2019). [PubMed: 30630850]

67. Palma M. et al. T cells in chronic lymphocytic leukemia display dysregulated expression of immune checkpoints and activation markers. Haematologica 102, 562-572, doi:10.3324/ haematol.2016.151100 (2017). [PubMed: 27927767]

68. Dancescu M. et al. Interleukin 4 protects chronic lymphocytic leukemic B cells from death by apoptosis and upregulates Bcl-2 expression. J Exp Med 176, 1319-1326, doi:10.1084/ jem.176.5.1319 (1992). [PubMed: 1402678]

69. Buschle M. et al. Interferon gamma inhibits apoptotic cell death in B cell chronic lymphocytic leukemia. J Exp Med 177, 213-218, doi:10.1084/jem.177.1.213 (1993). [PubMed: 7678114]

70. Kahan SM, Wherry EJ \& Zajac AJ T cell exhaustion during persistent viral infections. Virology 479-480, 180-193, doi:10.1016/j.virol.2014.12.033 (2015).

71. Ramsay AG et al. Chronic lymphocytic leukemia T cells show impaired immunological synapse formation that can be reversed with an immunomodulating drug. J Clin Invest 118, 2427-2437, doi:10.1172/JCI35017 (2008). [PubMed: 18551193]

72. Kabanova A. et al. Human Cytotoxic T Lymphocytes Form Dysfunctional Immune Synapses with B Cells Characterized by Non-Polarized Lytic Granule Release. Cell Rep 15, 9-18, doi:10.1016/ j.celrep.2016.02.084 (2016). [PubMed: 27052167]

73. Ramsay AG et al. Chronic lymphocytic leukemia cells induce defective LFA-1-directed T-cell motility by altering Rho GTPase signaling that is reversible with lenalidomide. Blood 121, 27042714, doi:10.1182/blood-2012-08-448332 (2013). [PubMed: 23325833]

74. Foa R. et al. Interleukin 2 (IL 2) and interferon-gamma production by $\mathrm{T}$ lymphocytes from patients with B-chronic lymphocytic leukemia: evidence that normally released IL 2 is absorbed by the neoplastic B cell population. Blood 66, 614-619 (1985). [PubMed: 2992637]

75. Burton J. \& Kay NE Does IL-2 receptor expression and secretion in chronic B-cell leukemia have a role in down-regulation of the immune system? Leukemia 8, 92-96 (1994). [PubMed: 8289505]

76. Wong KK, Brenneman F, Chesney A, Spaner DE \& Gorczynski RM Soluble CD200 is critical to engraft chronic lymphocytic leukemia cells in immunocompromised mice. Cancer Res 72, 49314943, doi:10.1158/0008-5472.CAN-12-1390 (2012). [PubMed: 22875025]

77. Ramsay AG, Clear AJ, Fatah R. \& Gribben JG Multiple inhibitory ligands induce impaired T-cell immunologic synapse function in chronic lymphocytic leukemia that can be blocked with lenalidomide: establishing a reversible immune evasion mechanism in human cancer. Blood 120, 1412-1421, doi:10.1182/blood-2012-02-411678 (2012). [PubMed: 22547582]

78. Brusa D. et al. The PD-1/PD-L1 axis contributes to T-cell dysfunction in chronic lymphocytic leukemia. Haematologica 98, 953-963, doi:10.3324/haematol.2012.077537 (2013). [PubMed: 23300177]

79. Grzywnowicz M. et al. Programmed death-1 and its ligand are novel immunotolerant molecules expressed on leukemic B cells in chronic lymphocytic leukemia. PLoS One 7, e35178, doi:10.1371/journal.pone.0035178 (2012).

80. Smith-Garvin JE, Koretzky GA \& Jordan MS T cell activation. Annu Rev Immunol 27, 591-619, doi:10.1146/annurev.immunol.021908.132706 (2009). [PubMed: 19132916] 
81. Feucht J. et al. T-cell responses against CD19+ pediatric acute lymphoblastic leukemia mediated by bispecific T-cell engager (BiTE) are regulated contrarily by PD-L1 and CD80/CD86 on leukemic blasts. Oncotarget 7, 76902-76919, doi:10.18632/oncotarget.12357 (2016).

82. Yellin MJ et al. T lymphocyte T cell-B cell-activating molecule/CD40-L molecules induce normal B cells or chronic lymphocytic leukemia B cells to express CD80 (B7/BB-1) and enhance their costimulatory activity. J Immunol 153, 666-674 (1994). [PubMed: 7517421]

83. Curran KJ et al. Enhancing antitumor efficacy of chimeric antigen receptor T cells through constitutive CD40L expression. Mol Ther 23, 769-778, doi:10.1038/mt.2015.4 (2015). [PubMed: 25582824]

84. Lin JK et al. Cost Effectiveness of Chimeric Antigen Receptor T-Cell Therapy in Multiply Relapsed or Refractory Adult Large B-Cell Lymphoma. J Clin Oncol 37, 2105-2119, doi:10.1200/ JCO.18.02079 (2019). [PubMed: 31157579]

85. Singh N. e. a. CAR T cell cytotoxicity is dependent on death receptor-driven apoptosis Blood 231, 698 (2018).

86. Lee L. et al. An APRIL-based chimeric antigen receptor for dual targeting of BCMA and TACI in multiple myeloma. Blood 131, 746-758, doi:10.1182/blood-2017-05-781351 (2018). [PubMed: 29284597]

87. Qin H. et al. Preclinical Development of Bivalent Chimeric Antigen Receptors Targeting Both CD19 and CD22. Mol Ther Oncolytics 11, 127-137, doi:10.1016/j.omto.2018.10.006 (2018). [PubMed: 30581986]

88. Ruella M. et al. Dual CD19 and CD123 targeting prevents antigen-loss relapses after CD19directed immunotherapies. J Clin Invest 126, 3814-3826, doi:10.1172/JCI87366 (2016). [PubMed: 27571406]

89. Mohammed S. et al. Improving Chimeric Antigen Receptor-Modified T Cell Function by Reversing the Immunosuppressive Tumor Microenvironment of Pancreatic Cancer. Mol Ther 25, 249-258, doi:10.1016/j.ymthe.2016.10.016 (2017). [PubMed: 28129119]

90. Mestermann K. et al. The tyrosine kinase inhibitor dasatinib acts as a pharmacologic on/off switch for CAR T cells. Sci Transl Med 11, doi:10.1126/scitranslmed.aau5907 (2019).

91. Li AM e. a. Checkpoint Inhibitors Augment CD19-Directed Chimeric Antigen Receptor (CAR) T Cell Therapy in Relapsed B-Cell Acute Lymphoblastic Leukemia. Blood 132, 556 (2018).

92. Chong EA e. a. Sequential Anti-CD19 Directed Chimeric Antigen Receptor Modified T-Cell Therapy (CART19) and PD-1 Blockade with Pembrolizumab in Patients with Relapsed or Refractory B-Cell Non-Hodgkin Lymphomas. Blood 132, 4198 (2018). 


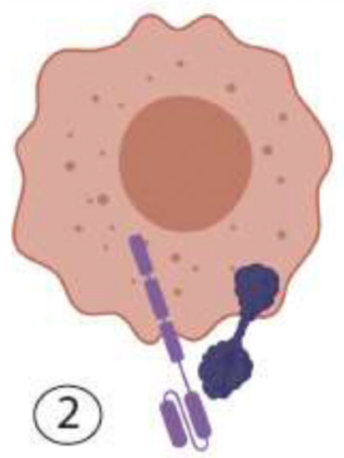

Mechanisms of tumor evasion

1. Antigen loss or reduction

2. Antigen masking

3. Suppressive microenvironment
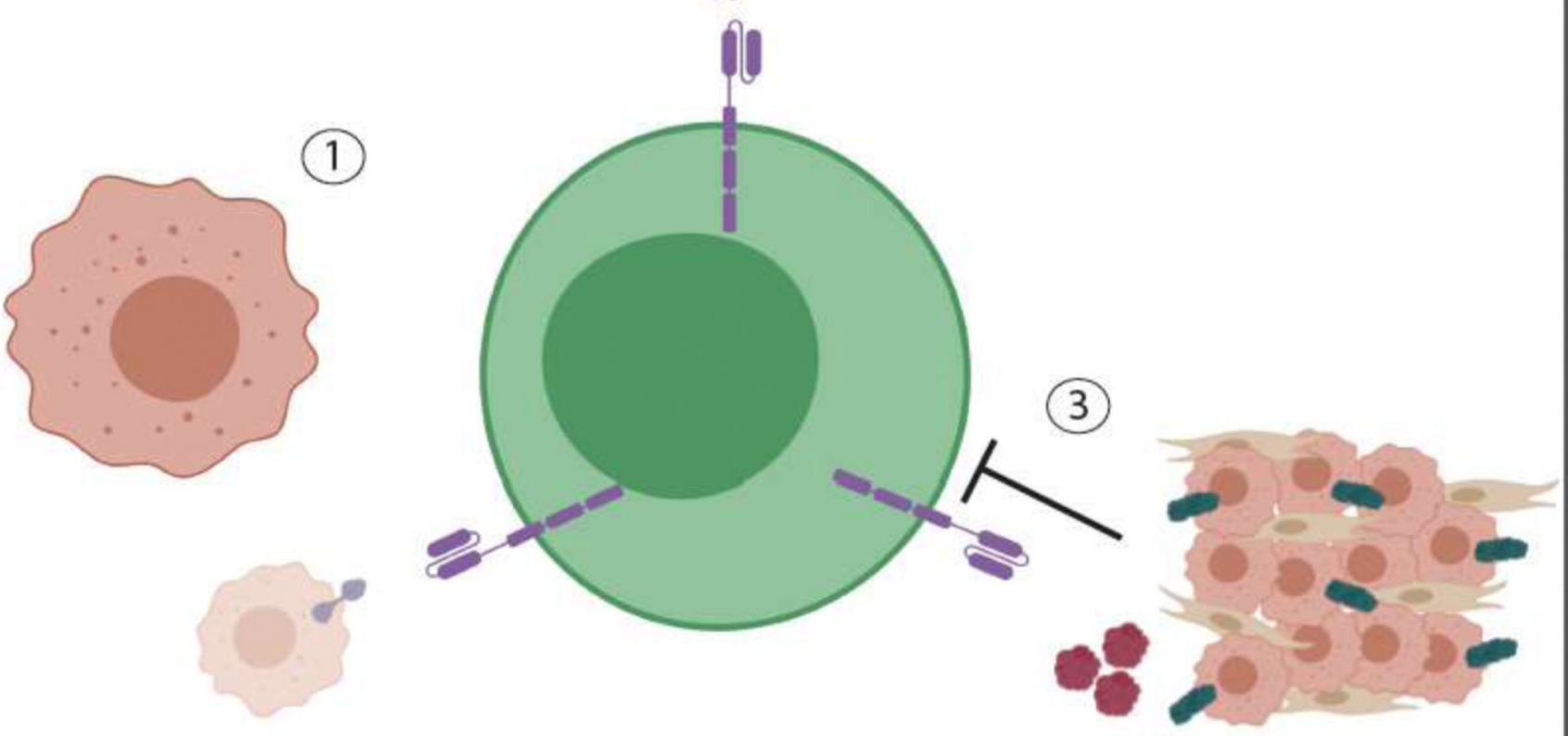

Figure 1. Identified mechanisms of tumor evasion to CAR $\mathbf{T}$ cells.

Summary of recent data of the cancer cell biology responsible for resisting CAR T cells (center, green): (1) loss or reduction of surface antigen expression, (2) masking of target antigen by co-expression of CAR and antigen on the leukemic cell surface, and (3) expression of immunosuppressive surface receptors and secreted factors. 


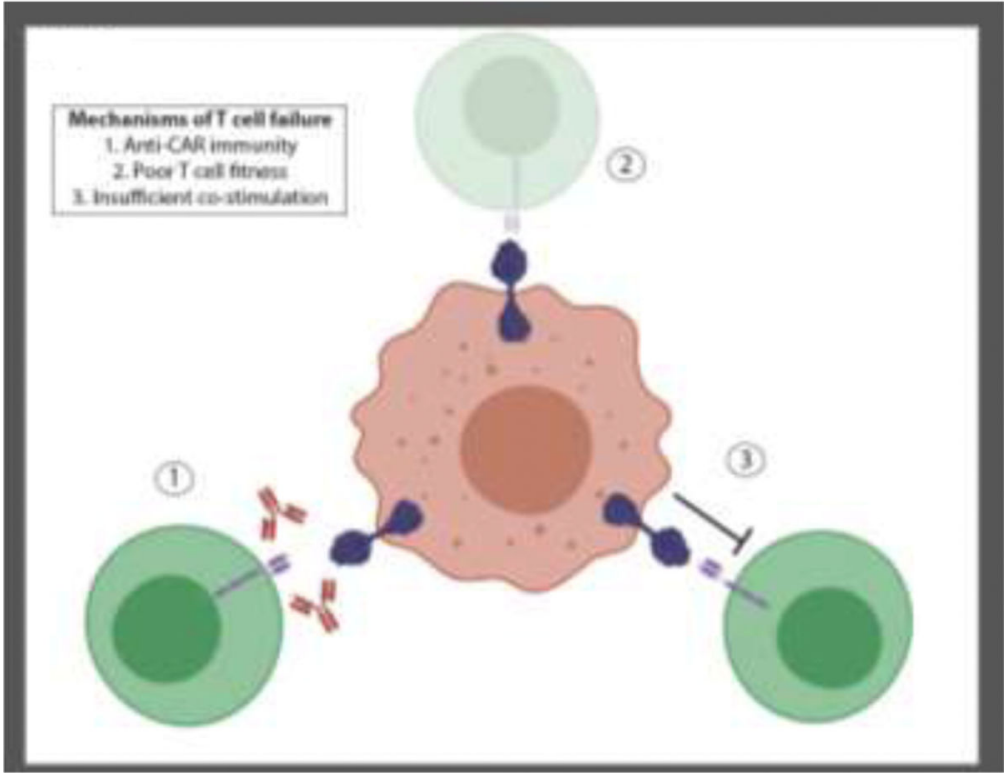

Figure 2. Identified mechanisms of $\mathbf{T}$ cell failure.

Summary of recent data on the $\mathrm{T}$ cell biology responsible for inherent resistance to CAR T cells. (1) anti-CAR immune responses mediated by the endogenous immune system, (2) low quality of the collected T cells used to manufacture the CAR product, and (3) ineffective costimulation of transferred $\mathrm{T}$ cells. 